\title{
A modified bordered construction for self-dual codes from group rings
}

\section{Joe Gildea, Abidin Kaya, Alexander Tylyshchak, Bahattin Yildiz}

\begin{abstract}
We describe a bordered construction for self-dual codes coming from group rings. We apply the constructions coming from the cyclic and dihedral groups over several alphabets to obtain extremal binary self-dual codes of various lengths. In particular we find a new extremal binary self-dual code of length 78 .
\end{abstract}

2010 MSC: 94B05

Keywords: Group rings, Self-dual codes, Codes over rings, Bordered constructions

\section{Introduction}

The standard form of the generator matrix of any binary self-dual code of length $2 n$ is of the form $\left(I_{n} \mid A\right)$ where $A$ is an $n \times n$ matrix satisfying $A A^{T}=-I_{n}$. When searching for self-dual codes, some special structure is imposed on the matrix $A$ to make the search field more feasible. Taking $A$ to be a circulant or a block circulant matrix is one of the methods that has been utilized in the literature. Sometimes, from a special such generator matrix, an extension can be achieved by modifying the matrix to get a new self-dual code of higher lengths. While these are generally known as extension methods in the literature, we can also view them as new construction methods for self-dual codes. One such example of a matrix, that we will extend in our constructions is defined in [13] as

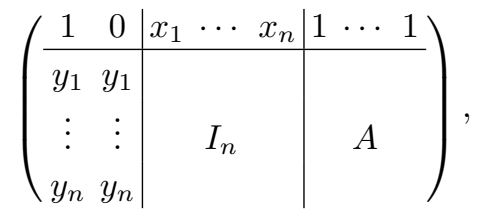

Joe Gildea; University of Chester, UK (email: J.Gildea@chester.ac.uk).

Abidin Kaya; Sampoerna University, Indonesia (email: nabidin@gmail.com).

Alexander Tylyshchak; Uzhgorod State University, Ukraine (email: alxtlk@gmail.com).

Bahattin Yildiz (Corresponding Author); Northern Arizona University, USA (email: bahattin.yildiz@nau.edu). 
where $y_{i}=x_{i}+1$ and $\left(I_{n} \mid A\right)$ is the generator matrix of a self-dual code of length $2 n$, possibly coming from a special construction method described above.

In this work, we shall extend the above construction, using matrices $A$ that arise from group rings. Group rings have been used to construct self-dual codes on many occasions. In [2], certain ideals of $\mathbb{F}_{2} S_{4}$ were used to construct the extended binary Golay code. In [15], an isomorphism from a group ring to a certain subring of the $n \times n$ matrices was described. This was used to construct self-dual codes in $[16,18,19]$. In [5], it is shown that zero divisors can't be used to construct the putative $[72,36,16]$ code. In [11], it is shown that unitary units can be used to construct self-dual codes under a certain construction, and using such units, many new extremal binary self-dual codes were obtained. In the same work, groups of different orders were used to describe many new construction methods for self-dual codes.

In our work, we will extend the structure of the matrix given in (1) with the matrices that we get from group ring elements to find new methods for constructing self-dual codes. We will apply the constructions coming from groups of order $2 p$ with $p$ an odd number (using the cyclic group $C_{2 p}$ and the Dihedral group $D_{2 p}$ ) over the binary field $\mathbb{F}_{2}, \mathbb{F}_{4}$ and the rings $R_{k}$ and $\mathbb{F}_{4}+u \mathbb{F}_{4}$ to obtain many extremal and best known binary self-dual codes of various lengths: $14,28,56,44,30,38,46,54,62,70$ and 78 . In particular, we obtain a new extremal binary self-dual code of length 78 . Many of these lengths are not well-known like the oft-studied lengths of 64,66 and 68.

The rest of the paper is organized as follows: In section 2 , we give some definitionas and notations that will be used in subsequent sections. In section 3 we give the construction together with a special case when it produces self-dual codes. In section 4 we give the computational results. We finish the paper with some concluding remarks and directions for possible future research.

\section{Definitions and notations}

\subsection{Codes} identity.

In this paper, all rings are assumed to be commutative, finite, Frobenius rings with a multiplicative

A code over a finite commutative ring $R$ is said to be any subset $C$ of $R^{n}$. When the code is a submodule of the ambient space then the code is said to be linear. To the ambient space, we attach the usual inner-product, specifically $[\mathbf{v}, \mathbf{w}]=\sum v_{i} w_{i}$. The orthogonal with respect to this inner-product is defined as $C^{\perp}=\left\{\mathbf{w} \mid \mathbf{w} \in R^{n},[\mathbf{v}, \mathbf{w}]=0, \forall \mathbf{v} \in C\right\}$. Since the ring is Frobenius we have that for all linear codes over $R,|C|\left|C^{\perp}\right|=|R|^{n}$.

If a code satisfies $C=C^{\perp}$ then the code $C$ is said to be self-dual. If $C \subseteq C^{\perp}$ then the code is said to be self-orthogonal. For binary codes, a self-dual code where all weights are congruent to $0(\bmod 4)$ is said to be Type II and the code is said to be Type I otherwise.

An upper bound on the minimum Hamming distance of a binary self-dual code finalized in [20].

Theorem 2.1. ([20/) Let $d_{I}(n)$ and $d_{I I}(n)$ be the minimum distance of a Type I and Type II binary code of length $n$, respectively. Then

$$
d_{I I}(n) \leq 4\left\lfloor\frac{n}{24}\right\rfloor+4
$$

and

$$
d_{I}(n) \leq \begin{cases}4\left\lfloor\frac{n}{24}\right\rfloor+4 & \text { if } n \neq 122 \quad(\bmod 24) \\ 4\left\lfloor\frac{n}{24}\right\rfloor+6 & \text { if } n \equiv 22 \quad(\bmod 24)\end{cases}
$$

Self-dual codes meeting these bounds are called extremal. A best known self-dual code of a given 
length is a self-dual code that has the highest possible known minimum distance, for a length for which the existence of an extremal code is not currently known.

Throughout the paper, we will be constructing extremal or best known binary self-dual codes of different lengths.

\subsection{Group rings and special matrices}

We shall use group rings in our construction so we give the standard definition of a group ring. Let $G$ be a finite group or order $n$, then the group $\operatorname{ring} R G$ consists of $\sum_{i=1}^{n} \alpha_{g_{i}} g_{i}, \alpha_{g_{i}} \in R, g_{i} \in G$.

Addition in the group ring is done by coordinate addition, namely

$$
\sum_{i=1}^{n} \alpha_{g_{i}} g_{i}+\sum_{i=1}^{n} \beta_{g_{i}} g_{i}=\sum_{i=1}^{n}\left(\alpha_{g_{i}}+\beta_{g_{i}}\right) g_{i}
$$

The product of two elements in a group ring is given by

$$
\left(\sum_{i=1}^{n} \alpha_{g_{i}} g_{i}\right)\left(\sum_{j=1}^{n} \beta_{g_{j}} g_{j}\right)=\sum_{i, j} \alpha_{g_{i}} \beta_{g_{j}} g_{i} g_{j}
$$

It follows that the coefficient of $g_{i}$ in the product is $\sum_{g_{i} g_{j}=g_{k}} \alpha_{g_{i}} \beta_{g_{j}}$.

We restrict ourselves to finite groups since we are mainly concerned with using these to construct codes whose lengths will be determined by the order of the group.

The following construction of a matrix was first given for codes over fields by Hurley in [15] and extended to rings in [5]. Let $R$ be a finite commutative Frobenius ring and let $G=\left\{g_{1}, g_{2}, \ldots, g_{n}\right\}$ be a group of order $n$. Let $v=\alpha_{g_{1}} g_{1}+\alpha_{g_{2}} g_{2}+\cdots+\alpha_{g_{n}} g_{n} \in R G$. Define the matrix $\sigma(v) \in M_{n}(R)$ to be

$$
\sigma(v)=\left(\begin{array}{ccccc}
\alpha_{g_{1}^{-1} g_{1}} & \alpha_{g_{1}^{-1} g_{2}} & \alpha_{g_{1}^{-1} g_{3}} & \ldots & \alpha_{g_{1}^{-1} g_{n}} \\
\alpha_{g_{2}^{-1} g_{1}} & \alpha_{g_{2}^{-1} g_{2}} & \alpha_{g_{2}^{-1} g_{3}} & \ldots & \alpha_{g_{2}^{-1} g_{n}} \\
\vdots & \vdots & \vdots & \vdots & \vdots \\
\alpha_{g_{n}^{-1} g_{1}} & \alpha_{g_{n}^{-1} g_{2}} & \alpha_{g_{n}^{-1} g_{3}} & \ldots & \alpha_{g_{n}^{-1} g_{n}}
\end{array}\right) .
$$

We note that the elements $g_{1}^{-1}, g_{2}^{-1}, \ldots, g_{n}^{-1}$ are the elements of the group $G$ in some given order.

Lemma 2.2. If $v=\sum_{i=1}^{n} \alpha_{g_{i}} g_{i}$ is unitary unit of $R G$ and $\mu=\sum_{i=1}^{n} \alpha_{g_{i}}$ then $\mu^{2}=1$.

Proof. The map $*: R G \rightarrow R G$ defined by $\left(\sum_{g \in G} a_{g} g\right)^{*}=\sum_{g \in G} a_{g} g^{-1}$ is an antiautomorphism of $R G$ of order 2. An element $v$ of $V(K G)$ satisfying $v v^{*}=1$ is called unitary. The homomorphism $\varepsilon: R G \rightarrow R$ given by $\varepsilon\left(\sum_{i=1}^{n} \alpha_{g_{i}} g_{i}\right)=\sum_{i=1}^{n} \alpha_{g_{i}}$ is called the augmentation mapping of $R G$. Let $v=\sum_{i=1}^{n} \alpha_{g_{i}} g_{i}$, then $v^{*}=\sum_{i=1}^{n} \alpha_{g_{i}} g_{i}^{-1}$ and $\varepsilon(v)=\varepsilon\left(v^{*}\right)=\sum_{i=1}^{n} \alpha_{g_{i}}=\mu$. Therefore $\varepsilon\left(v v^{*}\right)=\varepsilon(v) \varepsilon\left(v^{*}\right)=\mu^{2}=1$.

\subsection{1. $\sigma(v)$ for $D_{2 p}$ and $C_{2 p}$}

In what follows, $\operatorname{circ}\left(a_{1}, a_{2}, \ldots, a_{m}\right)$ denotes the $m \times m$ criculant matrix whose first row is given by $\left(a_{1}, a_{2}, \ldots, a_{m}\right)$. 
Let $G=C_{2 p}=\left\langle x \mid x^{2 p}=1\right\rangle$. If $\alpha=\sum_{i=0}^{p-1} \sum_{j=0}^{1} a_{1+i+p j} x^{2 i+j} \in R C_{2 p}$, then

$$
\sigma(\alpha)=\left(\begin{array}{cc}
A & B \\
B^{\prime} & A
\end{array}\right)
$$

where $A=\operatorname{circ}\left(a_{1}, \ldots, a_{p}\right), B=\operatorname{circ}\left(a_{p+1}, \ldots, a_{2 p}\right)$ and $B^{\prime}=\operatorname{circ}\left(a_{2 p}, a_{p+1}, \ldots, a_{2 p-1}\right)$.

Let $G=D_{2 p}=\left\langle x, y \mid x^{p}=y^{2}=1, x^{y}=x^{-1}\right\rangle$. If $\alpha=\sum_{i=0}^{p-1} \sum_{j=0}^{1} a_{1+i+p j} x^{i} y^{j} \in R D_{2 p}$, then

$$
\sigma(\alpha)=\left(\begin{array}{cc}
A & B \\
B^{T} & A^{T}
\end{array}\right)
$$

where $A=\operatorname{circ}\left(a_{1}, \ldots, a_{p}\right)$ and $B=\operatorname{circ}\left(a_{p+1}, \ldots, a_{2 p}\right)$, and $A^{T}$ represents the transpose of $A$.

\subsection{Rings}

We shall use several alphabets in our constructions, including the binary field $\mathbb{F}_{2}$, the quaternary field $\mathbb{F}_{4}$ and rings $R_{k}$ and $\mathbb{F}_{4}+u \mathbb{F}_{4}$.

\subsubsection{The ring family $R_{k}$}

The ring family $R_{k}$ were defined in [8] and [9]. We briefly give the descriptions of these rings.

For $k \geq 1$, define

$$
R_{k}=\mathbb{F}_{2}\left[u_{1}, u_{2}, \ldots, u_{k}\right] /\left\langle u_{i}^{2}=0, u_{i} u_{j}=u_{j} u_{i}\right\rangle
$$

For $k=1$, we denote the rings by $\mathbb{F}_{2}+u \mathbb{F}_{2}$, and when $k=2$, we denote them by $\mathbb{F}_{2}+u \mathbb{F}_{2}+v \mathbb{F}_{2}+u v \mathbb{F}_{2}$, both of which have been considered in coding theory quite extensively.

The rings can also be defined recursively as:

$$
R_{k}=R_{k-1}\left[u_{k}\right] /\left\langle u_{k}^{2}=0, u_{k} u_{j}=u_{j} u_{k}\right\rangle=R_{k-1}+u_{k} R_{k-1} .
$$

For any subset $A \subseteq\{1,2, \ldots, k\}$ we will fix

$$
u_{A}:=\prod_{i \in A} u_{i}
$$

with the convention that $u_{\emptyset}=1$. Then any element of $R_{k}$ can be represented as

$$
\sum_{A \subseteq\{1, \ldots, k\}} c_{A} u_{A}, \quad c_{A} \in \mathbb{F}_{2} .
$$

It is shown in [8] that the ring $R_{k}$ is a commutative ring with $\left|R_{k}\right|=2^{\left(2^{k}\right)}$. It is also shown that

$$
\forall a \in R_{k} \quad a^{2}= \begin{cases}1 & \text { if } a \text { is a unit } \\ 0 & \text { otherwise. }\end{cases}
$$


We shall now recall the Gray map from $R_{k}$ to $\mathbb{F}_{2}^{2^{k}}$. For $R_{1}$ we have the following map: $\phi_{1}(a+b u)=$ $(b, a+b)$. Then let $c \in R_{k}, c$ can be written as $c=a+b u_{k-1}, a, b \in R_{k-1}$. Then

$$
\phi_{k}(c)=\left(\phi_{k-1}(b), \phi_{k-1}(a+b)\right) \text {. }
$$

The map $\phi_{k}$ is a distance preserving map and the following is shown in [9].

Theorem 2.3. Let $C$ be a self-dual code over $R_{k}$, then $\phi_{k}\left(R_{k}\right)$ is a binary self-dual code of length $2^{k} n$.

\subsubsection{The ring $\mathbb{F}_{4}+u \mathbb{F}_{4}$}

Let $\mathbb{F}_{4}=\mathbb{F}_{2}(\omega)$ be the quadratic field extension of $\mathbb{F}_{2}$, where $\omega^{2}+\omega+1=0$. The ring $\mathbb{F}_{4}+u \mathbb{F}_{4}$ is defined via $u^{2}=0$. Note that $\mathbb{F}_{4}+u \mathbb{F}_{4}$ can be viewed as an extension of $R_{1}=\mathbb{F}_{2}+u \mathbb{F}_{2}$ and so we can describe any element of $\mathbb{F}_{4}+u \mathbb{F}_{4}$ in the form $\omega a+\bar{\omega} b$ uniquely, where $a, b \in \mathbb{F}_{2}+u \mathbb{F}_{2}$.

A linear code $C$ of length $n$ over $\mathbb{F}_{4}+u \mathbb{F}_{4}$ is an $\left(\mathbb{F}_{4}+u \mathbb{F}_{4}\right)$-submodule of $\left(\mathbb{F}_{4}+u \mathbb{F}_{4}\right)^{n}$. In [10] and [6] the following Gray maps were introduced;

$$
\begin{aligned}
& \psi_{\mathbb{F}_{4}}:\left(\mathbb{F}_{4}\right)^{n} \rightarrow\left(\mathbb{F}_{2}\right)^{2 n} \quad \| \varphi_{\mathbb{F}_{2}+u \mathbb{F}_{2}}:\left(\mathbb{F}_{2}+u \mathbb{F}_{2}\right)^{n} \rightarrow \mathbb{F}_{2}^{2 n} \\
& a \omega+b \bar{\omega} \mapsto(a, b), \quad a, b \in \mathbb{F}_{2}^{n} \| a+b u \mapsto(b, a+b), a, b \in \mathbb{F}_{2}^{n} .
\end{aligned}
$$

Those were generalized to the following maps in [17];

$$
\begin{aligned}
& \psi_{\mathbb{F}_{4}+u \mathbb{F}_{4}}:\left(\mathbb{F}_{4}+u \mathbb{F}_{4}\right)^{n} \rightarrow\left(\mathbb{F}_{2}+u \mathbb{F}_{2}\right)^{2 n} \\
& a \omega+b \bar{\omega} \mapsto(a, b), \quad a, b \in\left(\mathbb{F}_{2}+u \mathbb{F}_{2}\right)^{n}
\end{aligned} \| \begin{aligned}
& \varphi_{\mathbb{F}_{4}+u \mathbb{F}_{4}}:\left(\mathbb{F}_{4}+u \mathbb{F}_{4}\right)^{n} \rightarrow \mathbb{F}_{4}^{2 n} \\
& a+b u \mapsto(b, a+b), \quad a, b \in \mathbb{F}_{4}^{n}
\end{aligned}
$$

These maps preserve orthogonality in the corresponding alphabets. The binary images $\varphi_{\mathbb{F}_{2}+u \mathbb{F}_{2}} \circ$ $\psi_{\mathbb{F}_{4}+u \mathbb{F}_{4}}(C)$ and $\psi_{\mathbb{F}_{4}} \circ \varphi_{\mathbb{F}_{4}+u \mathbb{F}_{4}}(C)$ are equivalent. The Lee weight of an element is defined to be the Hamming weight of its binary image.

Proposition 2.4. ([17]) Let $C$ be a code over $\mathbb{F}_{4}+u \mathbb{F}_{4}$. If $C$ is self-orthogonal, so are $\psi_{\mathbb{F}_{4}+u \mathbb{F}_{4}}(C)$ and $\varphi_{\mathbb{F}_{4}+u \mathbb{F}_{4}}(C) . C$ is a Type $I$ (resp. Type II) code over $\mathbb{F}_{4}+u \mathbb{F}_{4}$ if and only if $\varphi_{\mathbb{F}_{4}+u \mathbb{F}_{4}}(C)$ is a Type I (resp. Type II) $\mathbb{F}_{4}$-code, if and only if $\psi_{\mathbb{F}_{4}+u \mathbb{F}_{4}}(C)$ is a Type $I$ (resp. Type II) $\mathbb{F}_{2}+u \mathbb{F}_{2}$-code. Furthermore, the minimum Lee weight of $C$ is the same as the minimum Lee weight of $\psi_{\mathbb{F}_{4}+u \mathbb{F}_{4}}(C)$ and $\varphi_{\mathbb{F}_{4}+u \mathbb{F}_{4}}(C)$.

Corollary 2.5. Suppose that $C$ is a self-dual code over $\mathbb{F}_{4}+u \mathbb{F}_{4}$ of length $n$ and minimum Lee distance d. Then $\varphi_{\mathbb{F}_{2}+u \mathbb{F}_{2}} \circ \psi_{\mathbb{F}_{4}+u \mathbb{F}_{4}}(C)$ is a binary $[4 n, 2 n, d]$ self-dual code. Moreover, $C$ and $\varphi_{\mathbb{F}_{2}+u \mathbb{F}_{2}} \circ \psi_{\mathbb{F}_{4}+u \mathbb{F}_{4}}(C)$ have the same weight enumerator. If $C$ is Type $I$ (Type II), then so is $\varphi_{\mathbb{F}_{2}+u \mathbb{F}_{2}} \circ \psi_{\mathbb{F}_{4}+u \mathbb{F}_{4}}(C)$.

\section{The construction}

Let $v \in R G$ where $R$ is a finite Frobenius ring of characteristic 2 and $G$ is a finite group of order $2 p$ and $p$ is odd. Define the following matrix:

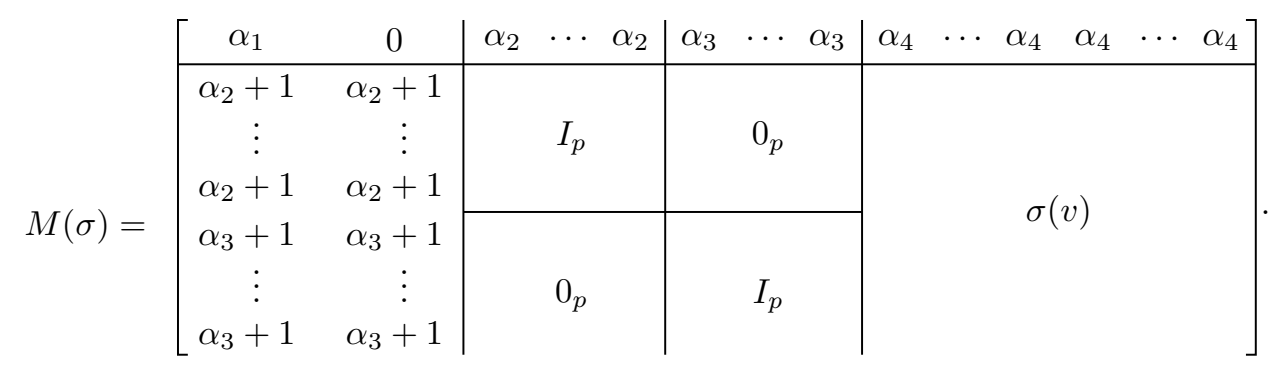


Let $C_{\sigma}$ be a code that is generated by the matrix $M(\sigma)$ and $\mu=\sum_{i=1}^{n} \alpha_{g_{i}}$. Let $A_{1}=\left(\alpha_{1}, 0\right) \in R^{2}$,

$$
\begin{gathered}
A_{2}=\left(\alpha_{2}, \ldots, \alpha_{2}, \alpha_{3}, \ldots, \alpha_{3}\right) \in R^{2 p}, A_{3}=\left(\alpha_{4}, \ldots, \alpha_{4}\right) \in R^{2 p} \text { and } B_{1}=\left(\begin{array}{cc}
\alpha_{2}+1 & \alpha_{2}+1 \\
\vdots & \vdots \\
\alpha_{2}+1 & \alpha_{2}+1 \\
\alpha_{3}+1 & \alpha_{3}+1 \\
\vdots & \vdots \\
\alpha_{3}+1 & \alpha_{3}+1
\end{array}\right) . \text { Then, } \\
M(\sigma)(M(\sigma))^{T}=\left(\begin{array}{cc}
A_{1} A_{1}^{T}+A_{2} A_{2}^{T}+A_{3} A_{3}^{T} & A_{1} B_{1}^{T}+A_{2}+A_{3} \sigma(v)^{T} \\
B_{1} A_{1}^{T}+A_{2}^{T}+\sigma(v) A_{3}^{T} & B_{1} B_{1}^{T}+I+\sigma(v) \sigma(v)^{T}
\end{array}\right)
\end{gathered}
$$

where

- $A_{1} A_{1}^{T}+A_{2} A_{2}^{T}+A_{3} A_{3}^{T}=\alpha_{1}^{2}+p \alpha_{2}^{2}+p \alpha_{3}^{2}+2 p \alpha_{4}^{2}=\alpha_{1}^{2}+p\left(\alpha_{2}+\alpha_{3}\right)^{2}$,

- $A_{1} B_{1}^{T}+A_{2}+A_{3} \sigma(v)^{T}=\left(\alpha_{1}\left(\alpha_{2}+1\right), \ldots, \alpha_{1}\left(\alpha_{2}+1\right), \alpha_{1}\left(\alpha_{3}+1\right), \ldots, \alpha_{1}\left(\alpha_{3}+1\right)\right)+$ $\left(\alpha_{2}, \ldots, \alpha_{2}, \alpha_{3}, \ldots, \alpha_{3}\right)+\left(\alpha_{4}, \ldots, \alpha_{4}\right) \sigma(v)=\left(\alpha_{1}\left(\alpha_{2}+1\right)+\alpha_{2}+\mu \alpha_{4}, \ldots, \alpha_{1}\left(\alpha_{2}+1\right)+\alpha_{2}+\mu \alpha_{4}, \alpha_{1}\left(\alpha_{3}+\right.\right.$ 1) $\left.+\alpha_{3}+\mu \alpha_{4}, \ldots, \alpha_{1}\left(\alpha_{3}+1\right)+\alpha_{3}+\mu \alpha_{4}\right)$ and

- $B_{1} B_{1}^{T}+I+\sigma(v) \sigma(v)^{T}=I+\sigma(v)\left(\sigma(v)^{T}=I+\sigma\left(v v^{*}\right)\right.$.

Theorem 3.1. Let $R$ be a finite commutative Frobenius ring of characteristic $2, G$ be a finite group of order $2 p$ where $p$ is odd and $\mu=\sum_{i=1}^{2 p} \alpha_{g_{i}}$. If $\alpha_{1}^{2}+p\left(\alpha_{2}+\alpha_{3}\right)^{2}=0, \alpha_{1}\left(\alpha_{2}+1\right)+\alpha_{2}+\mu \alpha_{4}=0$, $\alpha_{1}\left(\alpha_{3}+1\right)+\alpha_{3}+\mu \alpha_{4}=0 v v^{*}=1$ and $\left(\alpha_{1}, p\left(\alpha_{2}\left(\alpha_{2}+1\right)+\alpha_{3}\left(\alpha_{3}+1\right)\right), \alpha_{2}+1, \alpha_{3}+1\right)$ has free rank 1 then $C_{\sigma}$ is a self-dual code of length $4 p+2$.

Proof. If $v v^{*}=1$, then $I+\sigma\left(v v^{*}\right)=0$. Additionally, let $\alpha_{1}^{2}+p\left(\alpha_{2}+\alpha_{3}\right)^{2}=0, \alpha_{1}\left(\alpha_{2}+1\right)+\alpha_{2}+\mu \alpha_{4}=0$ and $\alpha_{1}\left(\alpha_{3}+1\right)+\alpha_{3}+\mu \alpha_{4}=0$, Therefore $C_{\sigma}$ is self-orthogonal. It remains to show that the $M(\sigma)$ has free rank $2 p+1$.

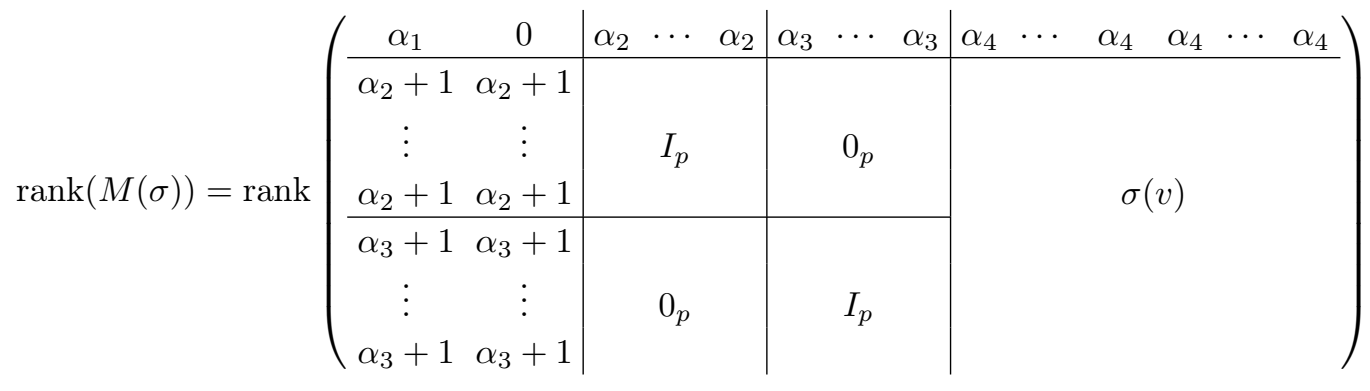

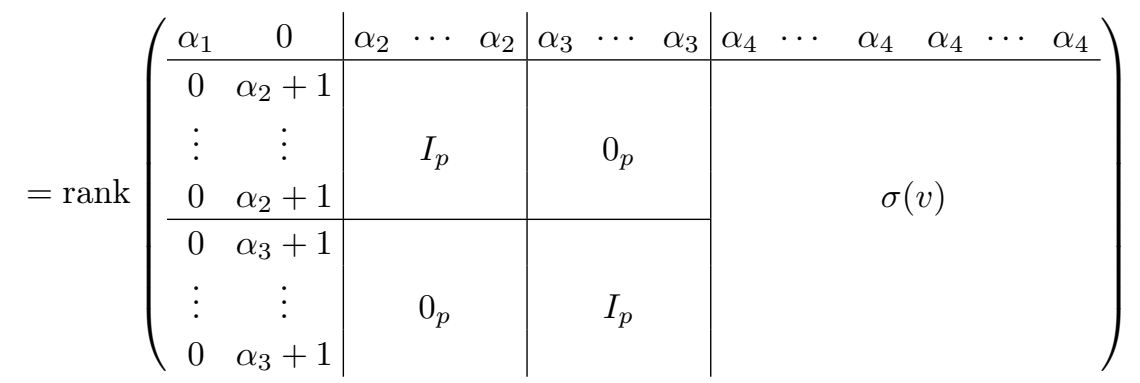



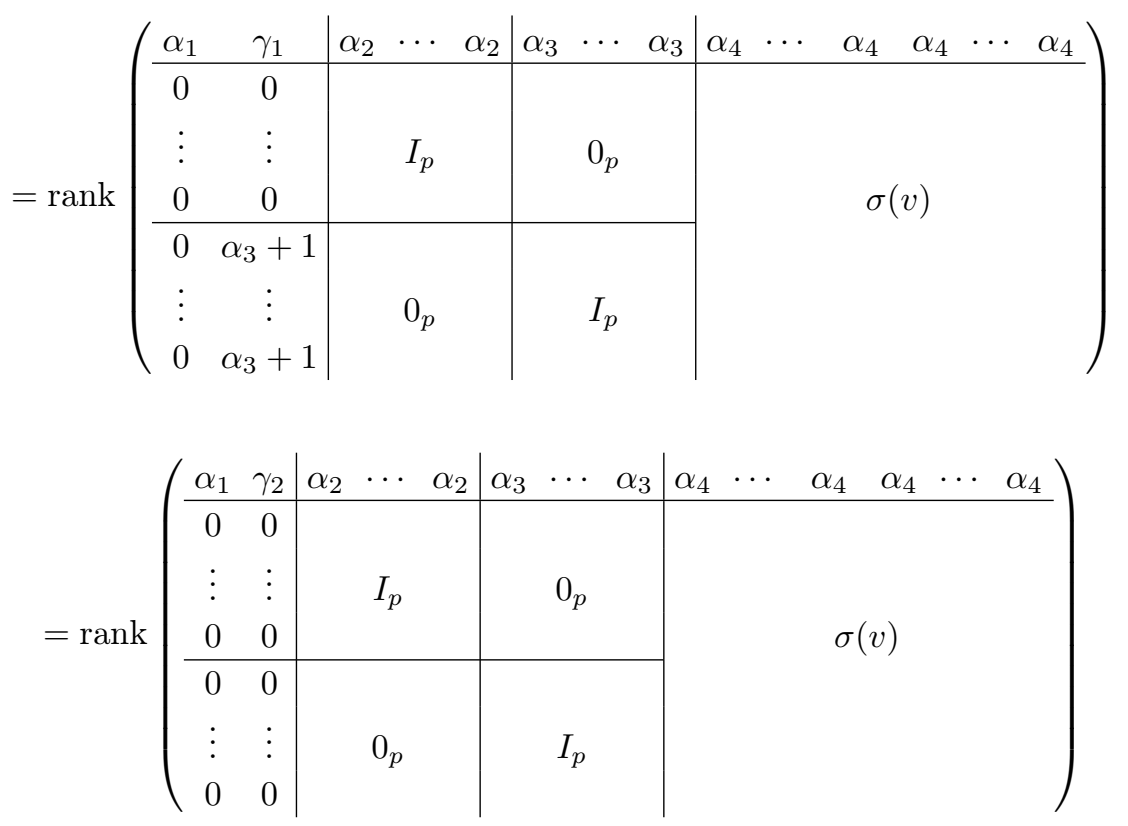

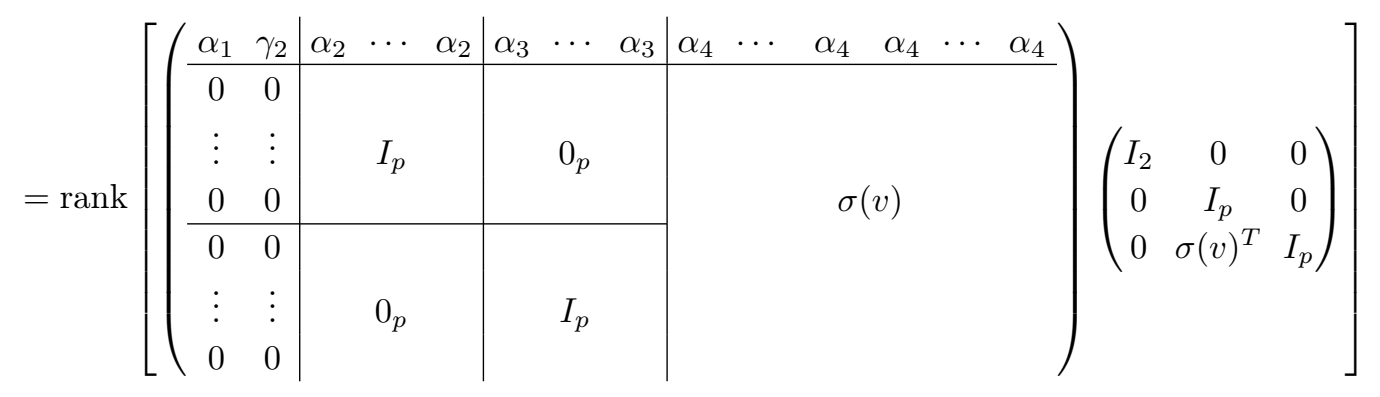

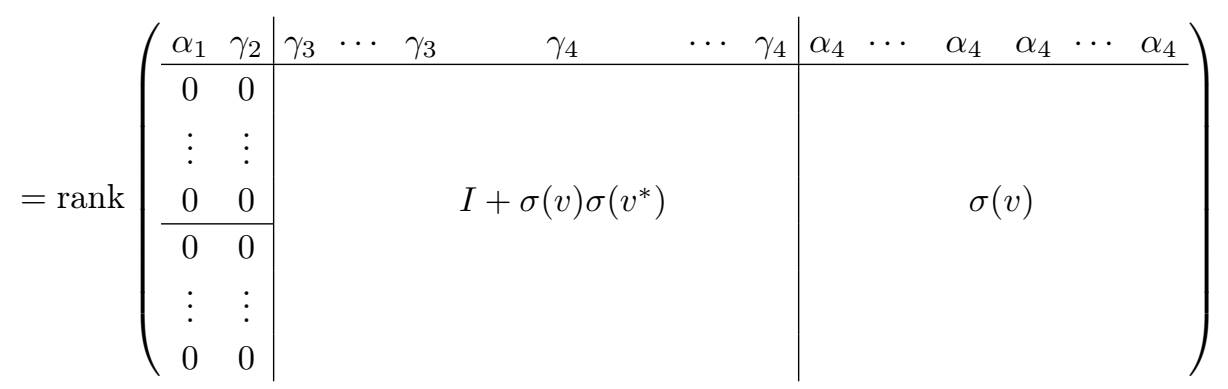

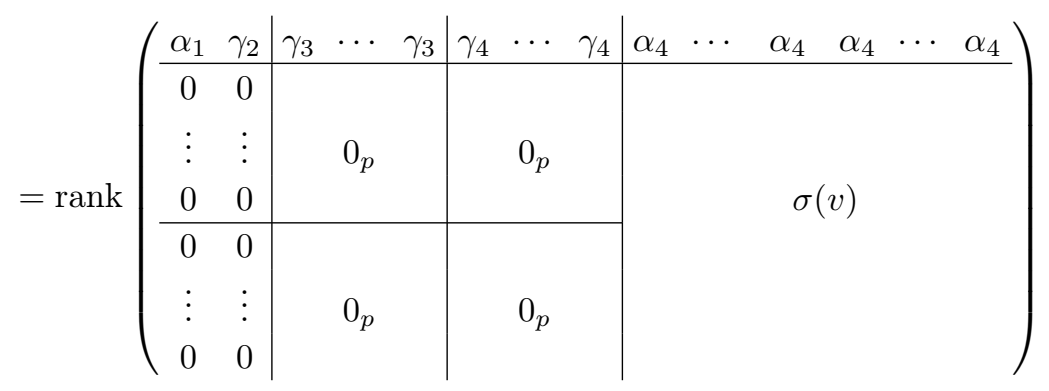


where $\gamma_{1}=p \alpha_{2}\left(\alpha_{2}+1\right), \gamma_{2}=p \alpha_{2}\left(\alpha_{2}+1\right)+p \alpha_{3}\left(\alpha_{3}+1\right), \gamma_{3}=\alpha_{2}+\mu \alpha_{4}=\alpha_{2}+1$ and $\gamma_{4}=\alpha_{3}+\mu \alpha_{4}=$ $\alpha_{3}+1$. Finally,

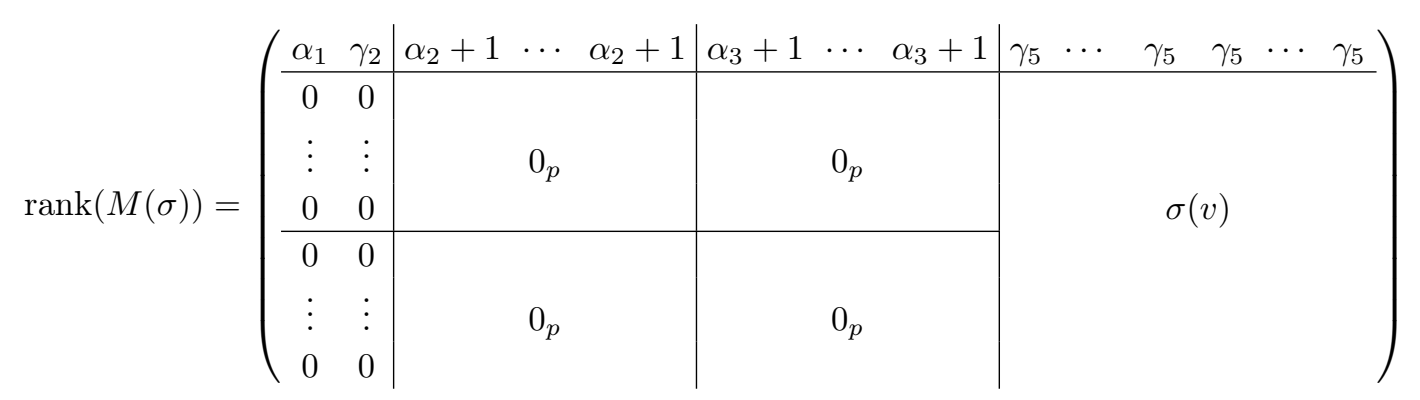

where $\gamma_{5}=\alpha_{4}+\mu^{2} \alpha_{4}=\alpha_{4}+(1) \alpha_{4}=0$ by Lemma 2.2. Therefore $C_{\sigma}$ is self if $\left(\alpha_{1}, p\left(\alpha_{2}\left(\alpha_{2}+1\right)+\alpha_{3}\left(\alpha_{3}+\right.\right.\right.$ 1)), $\left.\alpha_{2}+1, \alpha_{3}+1\right)$ has free rank 1 .

The family of rings $R_{k}$ is particularly well suited for this construction.

Corollary 3.2. Let $R=R_{k}$ and let $G$ be a finite group of order $2 p$ where $p$ is odd. Let $v \in R G$ be a unitary unit. Then if $\alpha_{2}+\alpha_{3}$ is any unit then $C_{\sigma}$ is a self-dual code of length $4 p+2$.

Proof. We will show that this case satisfies the hypotheses of Theorem 3.1. If $v$ is a unitary unit then $v v^{*}=1$. If $\alpha_{2}+\alpha_{3}$ is a unit then $\left(\alpha_{2}+\alpha_{3}\right)^{2}=1$ by (9). Then $1+p\left(\alpha_{2}+\alpha_{3}\right)^{2}=1+p(1)=0$ and $v v^{*}=1$.

\section{Computational results}

In this section, we apply the constructions discussed in the previous section over particular groups and rings that have been described before. The sizes of the groups and the alphabets used lead to particular lengths for self-dual codes. In all the subsequent subsections, we tabulate the extremal binary self-dual codes or the best-known (if the existence of extremal self-dual codes is not known for that length) self-dual codes of the certain lengths.

\subsection{Constructions from groups of order 6}

We first apply construction $D_{6}$ over the binary field and $\mathbb{F}_{4}$.

Table 1. Extremal binary self-dual code of length 14 from $D_{6}$

\begin{tabular}{ccc}
\hline$\left(\alpha_{1}, \alpha_{2}, \alpha_{3}, \alpha_{4}\right)$ & $\left(a_{1}, \ldots, a_{6}\right)$ & $|A u t(C)|$ \\
\hline$(1,0,1,1)$ & $(0,1,1,1,1,1)$ & $2^{7} \cdot 3^{2} \cdot 7^{2}$ \\
\hline
\end{tabular}

Table 2. Extremal self-dual code of length 28 coming from applying $D_{6}$ over $\mathbb{F}_{4}$

\begin{tabular}{lcc}
\hline$\left(\alpha_{1}, \alpha_{2}, \alpha_{3}, \alpha_{4}\right)$ & $\left(a_{1}, \ldots, a_{6}\right)$ & $|\operatorname{Aut}(C)|$ \\
\hline$(1, \omega, \omega+1,1)$ & $(0,0,1,1, \omega, \omega+1)$ & $2^{5} \cdot 3 \cdot 7$ \\
\hline
\end{tabular}


In [14], the possible weight enumerators for a self-dual Type I [56, 28, 10] code were obtained in two forms as:

$$
\begin{aligned}
& W_{56,1}=1+(308+4 \alpha) y^{10}+(4246-8 \alpha) y^{12}+(40852-28 \alpha) y^{14}+\cdots \\
& W_{56,2}=1+(308+4 \alpha) y^{10}+(3990-8 \alpha) y^{12}+(42900-28 \alpha) y^{14}+\cdots
\end{aligned}
$$

where $\alpha$ is an integer. Applying the constructions over the ring $\mathbb{F}_{4}+u \mathbb{F}_{4}$, we will be able to get binary self-dual codes of length 56 . For brevity of notation, we need a brief notation for the elements of $\mathbb{F}_{4}+u \mathbb{F}_{4}$.

$0 \leftrightarrow 0000,1 \leftrightarrow 0001,2 \leftrightarrow 0010,3 \leftrightarrow 0011,4 \leftrightarrow 0100,5 \leftrightarrow 0101,6 \leftrightarrow 0110,7 \leftrightarrow 0111,8 \leftrightarrow 1000$, $9 \leftrightarrow 1001, A \leftrightarrow 1010, B \leftrightarrow 1011, C \leftrightarrow 1100, D \leftrightarrow 1101, E \leftrightarrow 1110, F \leftrightarrow 1111$

We use the ordered basis $\{u \omega, \omega, u, 1\}$ to express the elements of $\mathbb{F}_{4}+u \mathbb{F}_{4}$. For instance, $1+u \omega$ corresponds to 1001, which is represented by the hexadecimal 9.

In the following tables $\alpha=m_{i}$ denotes that the weight enumerator of the code has parameter $\alpha=m$ in $W_{i}, i=1,2$.

Table 3. $[56,28,10]$ codes over $\mathbb{F}_{4}+u \mathbb{F}_{4}$ from $D_{6}$ where $\left(\alpha_{1}, \alpha_{2}, \alpha_{3}, \alpha_{4}\right)=(1,6, F, 1)$

\begin{tabular}{cccccc}
\hline$\left(a_{1}, \ldots, a_{6}\right)$ & $|A u t(C)|$ & Type & $\left(a_{1}, \ldots, a_{6}\right)$ & $|A u t(C)|$ & Type \\
\hline$(A, A, 1,1,4,5)$ & $2^{3} \cdot 3$ & $\alpha=-32_{2}$ & $(A, A, 1,3, C, F)$ & $2^{2} \cdot 3$ & $\alpha=-8_{2}$ \\
\hline$(A, A, 1, B, 6, D)$ & $2^{4} \cdot 3 \cdot 7$ & $\alpha=-56_{2}$ & $(A, 8,3,1,6,7)$ & $2 \cdot 3$ & $\alpha=-26_{2}$ \\
\hline$(A, 8,3,3, E, D)$ & $2 \cdot 3$ & $\alpha=-20_{2}$ & $(8,8,1,1,4,5)$ & $2^{4} \cdot 3$ & $\alpha=-8_{2}$ \\
\hline$(8,8,1,3, C, F)$ & $2^{2} \cdot 3$ & $\alpha=-32_{2}$ & & & \\
\hline
\end{tabular}

Table 4. $\quad[56,28,10]$ codes over $\mathbb{F}_{4}+u \mathbb{F}_{4}$ from $D_{6}$ where $|A u t(C)|=2 \cdot 3$

\begin{tabular}{cccccc}
\hline$\left(\alpha_{1}, \ldots, \alpha_{4}\right)$ & $\left(a_{1}, \ldots, a_{6}\right)$ & Type & $\left(a_{1}, \ldots, a_{6}\right)$ & $\left(\alpha_{1}, \ldots, \alpha_{4}\right)$ & Type \\
\hline$(1,4,7,1)$ & $(0,0,9,9, C, D)$ & $\alpha=-8_{2}$ & $(1,4,7,1)$ & $(0,8,3,1,4, F)$ & $\alpha=-24_{2}$ \\
\hline$(1,4,7,1)$ & $(0,0, B, 1, C, 7)$ & $\alpha=-18_{2}$ & $(1,4,7,1)$ & $(0,0, B, 3,4, D)$ & $\alpha=-12_{2}$ \\
\hline$(1,4,7,1)$ & $(2,0, B, 3, C, 7)$ & $\alpha=-20_{2}$ & $(1,4,7,1)$ & $(2,0, B, B, 6,5)$ & $\alpha=-14_{2}$ \\
\hline$(1,4,7,1)$ & $(2, A, 1,3,6, D)$ & $\alpha=-26_{2}$ & $(1,4,7,9)$ & $(A, 8,3,3, C, 7)$ & $\alpha=-6_{2}$ \\
\hline$(1,4,7,9)$ & $(8,2,9,3, E, 7)$ & $\alpha=-38_{2}$ & $(1,4,7,9)$ & $(8,2, B, 3,4, F)$ & $\alpha=-30_{2}$ \\
\hline$(1,4, D, 1)$ & $(0,8,1,1, E, 7)$ & $\alpha=-32_{2}$ & $(1,4, D, 1)$ & $(A, 0,9,9, E, 5)$ & $\alpha=-32_{1}$ \\
\hline$(1,4, D, 1)$ & $(A, 2, B, B, 4, D)$ & $\alpha=-26_{1}$ & $(1,4, D, 1)$ & $(A, A, 3,3,6,7)$ & $\alpha=-14_{1}$ \\
\hline$(1,4, D, 9)$ & $(0,0,9,1,4,5)$ & $\alpha=-26_{1}$ & $(1,4, D, 9)$ & $(A, 0,3,9,4, D)$ & $\alpha=-38_{1}$ \\
\hline$(1,4, F, 1)$ & $(2,8,9, B, 6, F)$ & $\alpha=-28_{1}$ & $(1,4, F, 1)$ & $(A, 8,1,1, C, F)$ & $\alpha=-22_{1}$ \\
\hline$(1,4, F, 9)$ & $(0,0,9,3, C, F)$ & $\alpha=-16_{1}$ & $(1,4, F, 9)$ & $(0,8,1, B, E, 5)$ & $\alpha=-28_{1}$ \\
\hline$(1,4, F, 9)$ & $(8,2,3,9,4, D)$ & $\alpha=-40_{1}$ & $(1,4, F, 9)$ & $(8,2,3, B, C, 7)$ & $\alpha=-46_{1}$ \\
\hline
\end{tabular}

We can also construct self-dual codes of length 56 from applying the construction $D_{6}$ over the ring $\mathbb{F}_{2}+u \mathbb{F}_{2}+v \mathbb{F}_{2}+u v \mathbb{F}_{2}$ as well. This is a ring of size 16 , so in the same way as was done for $\mathbb{F}_{4}+u \mathbb{F}_{4}$, we can use hexadecimals to shorten the notations. The correspondence between the binary 4 tuples and 
the hexadecimals is as follows:

$0 \leftrightarrow 0000,1 \leftrightarrow 0001,2 \leftrightarrow 0010,3 \leftrightarrow 0011,4 \leftrightarrow 0100,5 \leftrightarrow 0101,6 \leftrightarrow 0110,7 \leftrightarrow 0111,8 \leftrightarrow 1000$, $9 \leftrightarrow 1001, A \leftrightarrow 1010, B \leftrightarrow 1011, C \leftrightarrow 1100, D \leftrightarrow 1101, E \leftrightarrow 1110, F \leftrightarrow 1111$.

The ordered basis $\{u v, v, u, 1\}$ is used to express elements of $\mathbb{F}_{2}+u \mathbb{F}_{2}+v \mathbb{F}_{2}+u v \mathbb{F}_{2}$ For instance, $1+u+v$ is represented as 0111 which corresponds to hexadecimal 7.

Table 5. $[56,28,10]$ codes over $\mathbb{F}_{2}+u \mathbb{F}_{2}+v \mathbb{F}_{2}+u v \mathbb{F}_{2}$ from $D_{6}$ where $\left(\alpha_{1}, \alpha_{2}, \alpha_{3}, \alpha_{4}\right)=(1,6, B, 1)$

\begin{tabular}{cccccc}
\hline$\left(a_{1}, \ldots, a_{6}\right)$ & $\mid$ Aut $(C) \mid$ & Type & $\left(a_{1}, \ldots, a_{6}\right)$ & $\mid$ Aut $(C) \mid$ & Type \\
\hline$(4,1,9,1,7, B)$ & $2^{3} \cdot 3$ & $\alpha=-28_{2}$ & $(4,5, D, 1, F, 3)$ & $2^{3} \cdot 3$ & $\alpha=-52_{2}$ \\
\hline$(4,1,9,1, B, 7)$ & $2^{3} \cdot 3$ & $\alpha=-4_{2}$ & $(4,5, D, 9,7,3)$ & $2^{2} \cdot 3$ & $\alpha=-4_{2}$ \\
\hline$(4,5, D, 9,3,7)$ & $2^{2} \cdot 3$ & $\alpha=-16_{2}$ & $(4,7, F, 1,3, F)$ & $2^{2} \cdot 3$ & $\alpha=-28_{2}$ \\
\hline$(4,7, F, 9, F, B)$ & $2^{2} \cdot 3$ & $\alpha=-40_{2}$ & & & \\
\hline
\end{tabular}

Table 6. $[56,28,10]$ codes over $\mathbb{F}_{2}+u \mathbb{F}_{2}+v \mathbb{F}_{2}+u v \mathbb{F}_{2}$ from $D_{6}$ where $\left(\alpha_{1}, \alpha_{2}, \alpha_{3}, \alpha_{4}\right)=(1,2,5,1)$

\begin{tabular}{cccccc}
\hline$\left(a_{1}, \ldots, a_{6}\right)$ & $\mid$ Aut $(C) \mid$ & Type & $\left(a_{1}, \ldots, a_{6}\right)$ & $\mid$ Aut $(C) \mid$ & Type \\
\hline$(6,1,9,1, B, 5)$ & $2^{2} \cdot 3$ & $\alpha=-14_{1}$ & $(6,1,9,1, D, 3)$ & $2^{2} \cdot 3$ & $\alpha=-26_{1}$ \\
\hline$(0,1,9,5,3, F)$ & $2^{2} \cdot 3$ & $\alpha=-18_{1}$ & $(0,1,9,5,7, B)$ & $2^{2} \cdot 3$ & $\alpha=-42_{1}$ \\
\hline$(0,1,9,7, D, 3)$ & $2^{2} \cdot 3$ & $\alpha=-30_{1}$ & & & \\
\hline
\end{tabular}

Table 7. $[56,28,10]$ codes over $\mathbb{F}_{2}+u \mathbb{F}_{2}+v \mathbb{F}_{2}+u v \mathbb{F}_{2}$ from $D_{6}$ where $\left(\alpha_{1}, \alpha_{2}, \alpha_{3}, \alpha_{4}\right)=(1,2,7,1)$

\begin{tabular}{cccccc}
\hline$\left(a_{1}, \ldots, a_{6}\right)$ & $|A u t(C)|$ & Type & $\left(a_{1}, \ldots, a_{6}\right)$ & $|A u t(C)|$ & Type \\
\hline$(C, 7, F, 9,7, B)$ & $2^{2} \cdot 3$ & $\alpha=-38_{1}$ & $(C, 7, F, 9, B, 7)$ & $2^{2} \cdot 3 \cdot 13$ & $\alpha=-38_{1}$ \\
\hline
\end{tabular}

\subsection{Constructions from groups of order 10}

Table 8. Extremal binary self-dual code of length 22 from $D_{10}$

\begin{tabular}{ccc}
\hline$\left(\alpha_{1}, \alpha_{2}, \alpha_{3}, \alpha_{4}\right)$ & $\left(a_{1}, \ldots, a_{10}\right)$ & $|A u t(C)|$ \\
\hline$(1,0,1,1)$ & $(0,0,0,1,1,0,1,0,1,1)$ & $2^{8} \cdot 3^{2} \cdot 5 \cdot 7 \cdot 11$ \\
\hline
\end{tabular}

In [7], the possible weight enumerators for a self-dual Type I [44, 22, 8] code were obtained in two forms as:

$$
W_{44,1}=1+(44+4 \beta) y^{8}+(976-8 \beta) y^{10}+\cdots \text { where } 10 \leq \beta \leq 122 \text { and }
$$


$W_{44,2}=1+(44+4 \beta) y^{8}+(1232-8 \beta) y^{10}+\cdots$ where $10 \leq \beta \leq 154$

Table 9. Extremal self-dual code of length 44 over $\mathbb{F}_{4}$ from $D_{10}$

\begin{tabular}{lccc}
\hline$\left(\alpha_{1}, \alpha_{2}, \alpha_{3}, \alpha_{4}\right)$ & $\left(a_{1}, \ldots, a_{6}\right)$ & $|A u t(C)|$ & Type \\
\hline$(1, \omega, \omega+1,1)$ & $(0,0,0,1,1,1, \omega, \omega, \omega+1, \omega+1)$ & $2^{4} \cdot 5$ & $W_{44,2}(\beta=14)$ \\
\hline$(1, \omega, \omega+1,1)$ & $(0,0,0, \omega, \omega+1,0, \omega, \omega+1, \omega, \omega+1)$ & $2 \cdot 5$ & $W_{44,2}(\beta=4)$ \\
\hline$(1, \omega, \omega+1,1)$ & $(0,0,0, \omega, \omega+1,1,1, \omega, 1, \omega+1)$ & $2 \cdot 5$ & $W_{44,2}(\beta=4)$ \\
\hline$(1, \omega, \omega+1,1)$ & $(0,0,1,1,1,0, \omega, \omega, \omega+1, \omega+1)$ & $2^{3} \cdot 5$ & $W_{44,2}(\beta=34)$ \\
\hline$(1, \omega, \omega+1,1)$ & $(0,0,1,1,1,1,1,1, \omega, \omega+1)$ & $2^{3} \cdot 5$ & $W_{44,2}(\beta=34)$ \\
\hline$(1, \omega, \omega+1,1)$ & $(0,0,1, \omega, \omega+1,0,1,1, \omega+1, \omega)$ & 5 & $W_{44,2}(\beta=14)$ \\
\hline$(1, \omega, \omega+1,1)$ & $(0,0,1, \omega, \omega+1,0,1, \omega, 1, \omega+1)$ & 5 & $W_{44,2}(\beta=9)$ \\
\hline$(1, \omega, \omega+1,1)$ & $(0,0,1, \omega, \omega+1,1, \omega, \omega, \omega, \omega)$ & $2 \cdot 5$ & $W_{44,2}(\beta=4)$ \\
\hline$(1, \omega, \omega+1,1)$ & $(0,0, \omega, 1, \omega, 0,1,1, \omega+1, \omega+1)$ & $2^{2} \cdot 5$ & $W_{44,2}(\beta=4)$ \\
\hline$(1, \omega, \omega+1,1)$ & $(0,0, \omega, 1, \omega, 1, \omega, \omega, \omega, \omega+1)$ & $2 \cdot 5$ & $W_{44,2}(\beta=4)$ \\
\hline$(1, \omega, \omega+1,1)$ & $(0,0, \omega, 1, \omega+1,1,1,1,1,1)$ & $2^{16} \cdot 3^{2} \cdot 5^{2}$ & $W_{44,2}(\beta=74)$ \\
\hline$(1, \omega, \omega+1,1)$ & $(0,0,1, \omega, \omega, 1,0, \omega, \omega, \omega+1, \omega)$ & $2 \cdot 5$ & $W_{44,2}(\beta=4)$ \\
\hline$(1, \omega, \omega+1,1)$ & $(0, \omega, \omega, \omega+1, \omega+1,1, \omega, \omega+1, \omega, \omega+1)$ & $2 \cdot 5$ & $W_{44,2}(\beta=4)$ \\
\hline$(1, \omega, \omega+1,1)$ & $(1,1,1, \omega, \omega+1,1, \omega, \omega+1, \omega, \omega+1)$ & $2^{2} \cdot 5$ & $W_{44,2}(\beta=14)$ \\
\hline
\end{tabular}

Table 10. Extremal self-dual code of length 44 over $\mathbb{F}_{2}+u \mathbb{F}_{2}$ from $C_{10}$

\begin{tabular}{cccc}
\hline$\left(\alpha_{1}, \alpha_{2}, \alpha_{3}, \alpha_{4}\right)$ & $\left(a_{1}, \ldots, a_{6}\right)$ & $|A u t(C)|$ & Type \\
\hline$(1, u, 1,1)$ & $(u, u, u, 1,1,1,1,0, u+1,0)$ & $2^{2} \cdot 5$ & $W_{44,1}(\beta=32)$ \\
\hline$(1, u, 1,1)$ & $(u, u, u, 1,1, u+1, u+1,0, u+1,0)$ & $2^{15} \cdot 3^{4} \cdot 5^{2} \cdot 7^{2}$ & $W_{44,1}(\beta=122)$ \\
\hline$(1, u, 1,1)$ & $(u, 0, u, 1,1,1,1,0,1,0)$ & $2^{4} \cdot 5$ & $W_{44,2}(\beta=10)$ \\
\hline$(1, u, 1,1)$ & $(u, 0, u, 1,1, u+1, u+1,0,1,0)$ & $2^{3} \cdot 5$ & $W_{44,2}(\beta=30)$ \\
\hline$(1, u, 1,1)$ & $(0, u, 0,1,1, u+1, u+1, u, u+1, u)$ & $2^{3} \cdot 5$ & $W_{44,1}(\beta=12)$ \\
\hline$(1, u, 1,1)$ & $(0,0,0,1,1,1,1, u, 1, u)$ & $2^{16} \cdot 3^{2} \cdot 5^{2}$ & $W_{44,2}(\beta=90)$ \\
\hline$(1, u, u+1,1)$ & $(u, 0, u, 1,1,1,1,0,1,0)$ & $2^{4} \cdot 5$ & $W_{44,2}(\beta=14)$ \\
\hline$(1, u, u+1,1)$ & $(u, 0, u, 1,1, u+1, u+1,0,1,0)$ & $2^{3} \cdot 5$ & $W_{44,2}(\beta=34)$ \\
\hline$(1, u, u+1,1)$ & $(0,0,0,1,1,1,1, u, 1, u)$ & $2^{16} \cdot 3^{2} \cdot 5^{2}$ & $W_{44,2}(\beta=74)$ \\
\hline$(1,0, u+1,1)$ & $(u, 0, u, 1,1,1,1,0,1,0)$ & $2^{4} \cdot 5$ & $W_{44,2}(\beta=14)$ \\
\hline$(1,0, u+1,1)$ & $(u, 0, u, 1,1, u+1, u+1,0,1,0)$ & $2^{3} \cdot 5$ & $W_{44,2}(\beta=34)$ \\
\hline$(1,0, u+1,1)$ & $(0,0,0,1,1,1,1, u, 1, u)$ & $2^{16} \cdot 3^{4} \cdot 5^{2} \cdot 7^{2} \cdot 11^{2}$ & $W_{44,2}(\beta=154)$ \\
\hline
\end{tabular}




\subsection{Constructions from groups of order 14}

Table 11. Extremal binary self-dual code of length 30 from $D_{14}$

\begin{tabular}{ccc}
\hline$\left(\alpha_{1}, \alpha_{2}, \alpha_{3}, \alpha_{4}\right)$ & $\left(a_{1}, \ldots, a_{14}\right)$ & $\mid$ Aut $(C) \mid$ \\
\hline$(1,0,1,1)$ & $(0,0,0,0,0,0,1,0,0,1,0,1,1,1)$ & $2^{1} 1 \cdot 3 \cdot 7$ \\
\hline$(1,0,1,1)$ & $(0,0,0,0,0,1,1,0,0,1,0,0,1,1)$ & $2^{8} \cdot 7$ \\
\hline$(1,0,1,1)$ & $(0,0,0,1,0,1,1,0,1,1,1,1,1,1)$ & $2^{7} \cdot 3^{2} \cdot 5 \cdot 7$ \\
\hline
\end{tabular}

Table 12. Extremal binary self-dual code of length 30 from $C_{14}$

\begin{tabular}{ccc}
\hline$\left(\alpha_{1}, \alpha_{2}, \alpha_{3}, \alpha_{4}\right)$ & $\left(a_{1}, \ldots, a_{14}\right)$ & $|A u t(C)|$ \\
\hline$(1,0,1,1)$ & $(0,0,0,0,0,1,1,0,1,1,0,0,1,0)$ & $2^{8} \cdot 7$ \\
\hline
\end{tabular}

Table 13. Extremal binary self-dual code of length 60 over $\mathbb{F}_{4}$ from $D_{14}$

\begin{tabular}{|c|c|c|c|}
\hline$\left(\alpha_{1}, \alpha_{2}, \alpha_{3}, \alpha_{4}\right)$ & $\left(a_{1}, \ldots, a_{14}\right)$ & $|A u t(C)|$ & Type \\
\hline$(1, \omega, \omega+1,1)$ & $(0,0,1, \omega, 1, \omega, 1,0,1, \omega+1,0, \omega, \omega+1, \omega+1)$ & $2^{2} \cdot 7$ & $W_{60,1}(\beta=0)$ \\
\hline
\end{tabular}

\subsection{Constructions from groups of order 18}

Table 14. Extremal binary self-dual code of length 38 from $D_{18}$

\begin{tabular}{ccc}
\hline$\left(\alpha_{1}, \alpha_{2}, \alpha_{3}, \alpha_{4}\right)$ & $\left(a_{1}, \ldots, a_{18}\right)$ & $|A u t(C)|$ \\
\hline$(1,0,1,1)$ & $(0,0,0,0,0,0,1,1,1,0,0,1,1,0,1,1,1,1)$ & $2 \cdot 3^{2}$ \\
\hline$(1,0,1,1)$ & $(0,0,0,0,1,0,1,1,1,0,0,1,1,0,1,0,1,1)$ & $2 \cdot 3^{2} \cdot 19$ \\
\hline
\end{tabular}

\subsection{Constructions from groups of order 22}

Table 15. $[46,22,8]$ codes from $D_{22}$

\begin{tabular}{ccc}
\hline$\left(\alpha_{1}, \alpha_{2}, \alpha_{3}, \alpha_{4}\right)$ & $\left(a_{1}, \ldots, a_{22}\right)$ & $\mid$ Aut $(C) \mid$ \\
\hline$(1,0,1,1)$ & $(0,0,0,0,0,0,0,0,0,1,1,0,0,0,1,1,0,1,1,1,1,1)$ & $2 \cdot 11$ \\
\hline$(1,0,1,1)$ & $(0,0,0,0,0,0,0,0,1,1,1,0,0,1,0,1,1,0,1,0,1,1)$ & $2 \cdot 11$ \\
\hline$(1,0,1,1)$ & $(0,0,0,0,0,1,0,0,1,1,1,0,0,0,1,0,0,1,1,0,1,1)$ & 11 \\
\hline$(1,0,1,1)$ & $(0,0,0,1,0,1,1,0,1,1,1,1,1,1,1,1,1,1,1,1,1,1)$ & $2^{15} \cdot 3^{4} \cdot 5^{2} \cdot 7^{2} \cdot 11^{2} \cdot 23^{2}$ \\
\hline
\end{tabular}


Table 16. $[46,22,8]$ codes from $C_{22}$

\begin{tabular}{ccc}
\hline$\left(\alpha_{1}, \alpha_{2}, \alpha_{3}, \alpha_{4}\right)$ & $\left(a_{1}, \ldots, a_{22}\right)$ & $\mid$ Aut $(C) \mid$ \\
\hline$(1,0,1,1)$ & $(0,0,0,0,0,0,0,1,0,1,1,0,1,1,1,0,1,1,0,0,0,1)$ & $2 \cdot 11$ \\
\hline$(1,0,1,1)$ & $(0,0,0,0,0,0,1,0,1,1,1,0,0,1,1,0,1,1,0,1,0,0)$ & $2 \cdot 11$ \\
\hline$(1,0,1,1)$ & $(0,0,0,0,1,0,1,1,1,1,1,0,1,1,0,1,1,1,1,0,1,0)$ & $2 \cdot 11$ \\
\hline$(1,0,1,1)$ & $(0,0,0,1,0,0,1,1,1,1,1,1,1,0,1,1,0,1,1,0,0,1)$ & $2 \cdot 11$ \\
\hline$(1,0,1,1)$ & $(0,0,0,0,1,0,1,1,0,1,1,1,1,1,1,1,1,1,1,1,1,1,1)$ & $2^{15} \cdot 3^{4} \cdot 5^{2} \cdot 7^{2} \cdot 11^{2} \cdot 23^{2}$ \\
\hline
\end{tabular}

\subsection{Constructions from groups of order 26}

From [3], it is known that the weight enumerator of a $[54,27,10]$ self-dual code can be of the follwing form:

$$
\begin{aligned}
& W_{54,1}=1+(351-8 \beta) y^{10}+(5031+24 \beta) y^{12}+\ldots \\
& W_{54,2}=1+(351-8 \beta) y^{10}+(5543+24 \beta) y^{12}+\ldots
\end{aligned}
$$

In the following tables, we consturct inequivalent self-dual codes of parameters $[54,27,10]$ from $D_{26}$ and $C_{26}$.

Table 17. Inequivalent $[54,27,10]$ codes from $D_{26}$

\begin{tabular}{ccccc}
\hline$\left(\alpha_{1}, \alpha_{2}, \alpha_{3}, \alpha_{4}\right)$ & $\left(a_{1}, \ldots, a_{26}\right)$ & $\mid$ Aut $(C) \mid$ & Type \\
\hline$(1,0,1,1)$ & $(0,0,0,0,0,0,0,0,0,0,0,1,1,0,0,0,1,0,1,1,0,1,1,1,0,1)$ & $2 \cdot 13$ & $W_{54,1}(\beta=0)$ \\
\hline$(1,0,1,1)$ & $(0,0,0,0,0,0,0,0,0,0,0,1,1,0,0,0,1,1,0,1,0,1,0,1,1,1)$ & $2 \cdot 13$ & $W_{54,1}(\beta=0)$ \\
\hline$(1,0,1,1)$ & $(0,0,0,0,0,0,0,0,0,1,0,1,1,0,0,1,0,1,0,0,1,0,1,0,1,1)$ & 13 & $W_{54,1}(\beta=0)$ \\
\hline$(1,0,1,1)$ & $(0,0,0,0,0,0,0,0,1,0,0,1,1,0,0,0,1,0,0,1,1,1,1,0,0,1)$ & $2 \cdot 3 \cdot 13$ & $W_{54,1}(\beta=0)$ \\
\hline$(1,0,1,1)$ & $(0,0,0,0,0,0,0,1,0,1,0,1,1,0,0,0,0,0,1,0,0,0,1,1,1,1)$ & 13 & $W_{54,1}(\beta=0)$ \\
\hline$(1,0,1,1)$ & $(0,0,0,0,1,0,0,1,1,0,1,1,1,0,0,1,0,1,0,1,0,0,1,1,1,1)$ & $2 \cdot 3^{4} \cdot 13$ & $W_{54,1}(\beta=0)$ \\
\hline$(1,0,1,1)$ & $(0,0,0,0,1,1,0,1,0,1,0,1,1,0,0,0,1,0,0,1,1,0,1,1,1,1)$ & $2 \cdot 3 \cdot 13$ & $W_{54,1}(\beta=0)$ \\
\hline
\end{tabular}

Table 18. Inequivalent $[54,27,10]$ codes from $C_{26}$

\begin{tabular}{cccc}
\hline$\left(\alpha_{1}, \alpha_{2}, \alpha_{3}, \alpha_{4}\right)$ & $\left(a_{1}, \ldots, a_{26}\right)$ & $|A u t(C)|$ & Type \\
\hline$(1,0,1,1)$ & $(0,0,0,0,0,0,0,0,0,1,0,1,1,0,1,0,1,0,0,1,0,1,0,1,1,0)$ & $2 \cdot 13$ & $W_{54,1}(\beta=0)$ \\
\hline$(1,0,1,1)$ & $(0,0,0,0,0,0,0,0,1,0,0,1,1,0,0,0,1,0,0,1,1,0,1,1,1,0)$ & $2 \cdot 3 \cdot 13$ & $W_{54,1}(\beta=0)$ \\
\hline$(1,0,1,1)$ & $(0,0,0,0,0,0,0,0,1,0,1,1,1,0,1,1,0,1,0,0,1,1,1,1,1,1)$ & $2 \cdot 13$ & $W_{54,1}(\beta=0)$ \\
\hline$(1,0,1,1)$ & $(0,0,0,0,0,0,0,1,0,1,0,1,1,1,1,0,1,1,0,1,1,1,1,0,1,0)$ & $2 \cdot 13$ & $W_{54,1}(\beta=0)$ \\
\hline$(1,0,1,1)$ & $(0,0,0,0,0,0,1,0,1,0,1,1,1,1,1,0,0,1,0,1,1,1,1,0,1,0)$ & $2 \cdot 13$ & $W_{54,1}(\beta=0)$ \\
\hline$(1,0,1,1)$ & $(0,0,0,0,0,0,1,0,1,1,1,1,1,1,1,0,0,0,0,1,0,1,1,1,0,1)$ & $2 \cdot 13$ & $W_{54,1}(\beta=0)$ \\
\hline$(1,0,1,1)$ & $(0,0,0,0,0,1,0,0,1,1,1,0,1,1,0,1,1,1,0,0,1,0,1,1,0,1)$ & $2 \cdot 13$ & $W_{54,1}(\beta=0)$ \\
\hline$(1,0,1,1)$ & $(0,0,0,0,0,1,0,1,1,1,1,1,1,1,0,0,0,1,0,1,1,1,1,0,0,0)$ & $2 \cdot 13$ & $W_{54,1}(\beta=0)$ \\
\hline$(1,0,1,1)$ & $(0,0,0,0,0,1,1,0,1,1,1,1,1,1,0,1,1,0,1,1,1,1,1,1,1,0)$ & $2 \cdot 13$ & $W_{54,1}(\beta=0)$ \\
\hline$(1,0,1,1)$ & $(0,0,0,1,0,0,1,1,0,1,1,1,1,0,1,1,0,1,1,1,1,1,1,1,1,0)$ & $2 \cdot 3 \cdot 13$ & $W_{54,1}(\beta=0)$ \\
\hline
\end{tabular}




\subsection{Constructions from groups of order 30}

There are two possibilities for the weight enumerators of extremal singly-even $[62,31,12]_{2}$ codes ([3]):

$$
\begin{aligned}
& W_{62,1}=1+2308 y^{12}+23767 y^{14}+\cdots \\
& W_{62,2}=1+(1860+32 \beta) y^{12}+(28055-160 \beta) y^{14}+\cdots,: 0 \leq \beta \leq 93 .
\end{aligned}
$$

only codes with weight enumerator for $\beta=0,2,9,10,15,16$ in $W_{62,2}$ known to exist.

Table 19. $[62,31,12]$ codes from $D_{30}$ where $\left(\alpha_{1}, \alpha_{2}, \alpha_{3}, \alpha_{4}\right)=(1,0,1,1)$

\begin{tabular}{ccc}
\hline$\left(a_{1}, \ldots, a_{30}\right)$ & $|A u t(C)|$ & Type \\
\hline$(0,0,0,0,0,0,0,0,1,0,1,1,1,0,1,0,0,1,0,1,1,0,1,0,1,0,1,0,1,1)$ & $2 \cdot 3 \cdot 5$ & $W_{62,2}(\beta=10)$ \\
\hline$(0,0,0,0,0,0,0,0,1,0,1,1,1,1,1,0,0,1,0,0,1,0,1,1,0,0,1,1,0,1)$ & $2 \cdot 3 \cdot 5$ & $W_{62,2}(\beta=10)$ \\
\hline$(0,0,0,0,0,0,1,0,0,1,0,0,1,0,1,0,0,0,0,1,1,1,0,1,1,1,0,1,1,1)$ & $22 \cdot 3 \cdot 5$ & $W_{62,2}(\beta=0)$ \\
\hline$(0,0,0,0,0,0,1,0,0,1,0,1,1,1,1,0,0,1,1,1,1,0,0,1,1,1,1,1,1,1)$ & $2 \cdot 3 \cdot 5$ & $W_{62,2}(\beta=0)$ \\
\hline$(0,0,0,0,0,1,0,0,1,0,0,1,1,1,1,0,1,0,1,1,0,1,1,1,1,1,1,0,1,1)$ & $2 \cdot 3 \cdot 5$ & $W_{62,2}(\beta=10)$ \\
\hline$(0,0,0,0,0,1,1,0,0,1,0,1,1,1,1,0,0,1,1,0,1,1,1,0,1,1,0,1,1,1)$ & $3 \cdot 5$ & $W_{62,2}(\beta=0)$ \\
\hline$(0,0,0,0,0,1,1,0,1,1,0,1,1,1,1,0,0,1,0,1,0,1,0,0,1,1,1,1,1,1)$ & $2 \cdot 3 \cdot 5$ & $W_{62,2}(\beta=0)$ \\
\hline$(0,0,0,0,0,1,1,1,0,1,0,1,1,1,1,0,1,1,1,1,1,0,1,1,1,1,1,1,1,1)$ & $2 \cdot 3 \cdot 5$ & $W_{62,2}(\beta=0)$ \\
\hline$(0,0,0,0,1,1,0,0,1,1,1,1,0,1,1,0,1,0,1,0,1,1,0,1,0,1,0,1,1,1)$ & $2 \cdot 3 \cdot 5$ & $W_{62,2}(\beta=0)$ \\
\hline
\end{tabular}

\subsection{Constructions from groups of order 34}

The weight enumerator of a self-dual $[70,35,12]_{2}$ code is in one of the following forms ([13]):

$$
\begin{aligned}
& W_{70,1}=1+2 \beta+(11730-2 \beta-128 \gamma) y^{14}+(150535-22 \beta+896 \gamma) y^{16}+\cdots \\
& W_{70,2}=1+2 \beta+(9682-2 \beta) y^{14}+(173063-22 \beta) y^{16}+\cdots
\end{aligned}
$$

The code with weight enumerator for $\gamma=1, \beta=416$ is constructed in [13]. Together with the results from [4] and [12], the existence of codes with weight enumerators $\gamma=0$ in $W_{70,1}$ is known for many $\beta$ values. In the following tables we tabulate the $[70,35,12]$ self-dual codes from $D_{34}$ and $C_{34}$ together with their $\beta$ values and automorphism groups. Note that the automorphism groups all have an element of order 17 in them. Naturally, these have the same parameters as the ones obtained in [12]. However, here we have given an alternative construction to those codes. 
Table 20. $[70,35,12]$ codes from $D_{34}$ where $\left(\alpha_{1}, \alpha_{2}, \alpha_{3}, \alpha_{4}\right)=(1,0,1,1)$

\begin{tabular}{ccc}
\hline$\left(a_{1}, \ldots, a_{34}\right)$ & $|A u t(C)|$ & $W_{70,1}(\gamma=0)$ \\
\hline$(0,0,0,0,0,0,0,0,1,0,1,0,1,1,0,0,1,0,0,0,0,0,1,1,0,1,0,1,1,0,1,0,1,1)$ & $2 \cdot 17$ & $\beta=102$ \\
\hline$(0,0,0,0,0,0,0,0,0,0,1,0,1,1,1,0,1,0,0,0,0,0,1,0,1,1,0,0,1,1,0,1,1,1)$ & $2 \cdot 17$ & $\beta=136$ \\
\hline$(0,0,0,0,0,0,0,0,1,0,1,0,1,1,1,0,1,0,0,0,1,0,0,0,1,0,0,1,0,1,1,0,1,1)$ & 17 & $\beta=170$ \\
\hline$(0,0,0,0,0,0,0,0,1,0,1,0,1,1,0,0,1,0,0,1,0,0,1,0,1,0,0,1,1,0,0,1,1,1)$ & 17 & $\beta=204$ \\
\hline$(0,0,0,0,0,0,0,0,1,0,1,0,1,1,0,0,0,0,0,1,0,0,1,0,1,1,0,1,1,0,1,0,1,1)$ & 17 & $\beta=238$ \\
\hline$(0,0,0,0,0,0,0,0,1,0,1,0,1,1,0,0,0,0,0,1,0,1,1,0,1,0,1,0,1,1,0,0,1,1)$ & 17 & $\beta=272$ \\
\hline$(0,0,0,0,0,0,0,0,1,0,1,0,1,1,0,0,0,0,0,0,1,0,0,0,1,1,0,1,1,0,1,1,1,1)$ & 17 & $\beta=306$ \\
\hline$(0,0,0,0,0,0,0,0,1,0,1,0,1,1,0,1,0,0,0,0,1,0,1,0,1,0,0,1,1,0,0,1,1,1)$ & 17 & $\beta=340$ \\
\hline$(0,0,0,0,0,0,0,0,1,0,1,0,1,1,0,1,1,0,0,0,0,0,0,1,0,1,1,1,0,0,0,1,1,1)$ & 17 & $\beta=374$ \\
\hline$(0,0,0,0,0,0,0,0,1,0,1,0,1,1,0,0,1,0,0,0,0,1,0,1,0,0,1,0,1,1,0,1,1,1)$ & 17 & $\beta=408$ \\
\hline$(0,0,0,0,0,0,0,0,1,0,1,0,1,1,0,0,1,0,0,0,1,0,1,0,1,1,0,0,0,1,0,1,1,1)$ & 17 & $\beta=442$ \\
\hline$(0,0,0,0,0,0,0,0,1,1,0,1,1,1,1,1,1,0,0,1,0,1,1,0,0,1,1,0,1,0,0,1,1,1)$ & $2 \cdot 17$ & $\beta=476$ \\
\hline$(0,0,0,0,0,0,0,0,1,1,1,1,0,1,0,0,0,0,0,0,0,1,0,1,0,1,0,1,0,0,1,1,1,1)$ & 17 & $\beta=510$ \\
\hline
\end{tabular}

Table 21. Extremal $[70,35,12]$ codes from $C_{34}$ where $\left(\alpha_{1}, \alpha_{2}, \alpha_{3}, \alpha_{4}\right)=(1,0,1,1)$

\begin{tabular}{ccc}
\hline$\left(a_{1}, \ldots, a_{34}\right)$ & $\mid$ Aut $(C) \mid$ & $W_{70,1}(\gamma=0)$ \\
\hline$(0,0,0,0,0,0,0,0,1,0,1,1,0,0,0,1,1,0,1,0,0,1,0,1,0,1,1,0,1,0,0,1,1,0)$ & $2 \cdot 17$ & $\beta=102$ \\
\hline$(0,0,0,0,0,0,0,1,0,1,1,0,1,1,1,1,1,0,0,0,0,1,0,1,1,0,0,1,0,1,1,1,1,1)$ & $2 \cdot 17$ & $\beta=136$ \\
\hline$(0,0,0,0,0,0,0,0,0,1,0,1,1,1,0,1,1,0,0,0,1,0,1,1,0,0,0,1,1,0,0,0,1,1)$ & $2 \cdot 17$ & $\beta=238$ \\
\hline$(0,0,0,0,0,0,1,0,0,1,1,1,0,1,1,0,1,1,1,1,1,0,0,0,0,0,1,1,0,1,0,1,1,1)$ & $2 \cdot 17$ & $\beta=272$ \\
\hline$(0,0,0,0,0,0,0,1,0,1,0,1,1,1,1,1,1,1,1,1,1,1,1,0,1,1,0,1,1,1,0,1,0,1)$ & $2 \cdot 17$ & $\beta=306$ \\
\hline$(0,0,0,0,0,0,0,0,0,0,0,0,1,0,0,1,1,0,0,1,1,0,1,1,0,1,1,1,0,1,0,0,1,1)$ & $2 \cdot 17$ & $\beta=374$ \\
\hline$(0,0,0,0,0,0,1,0,0,0,1,1,1,1,1,0,1,1,0,1,1,0,1,0,0,0,0,1,1,0,1,1,1,1)$ & $2 \cdot 17$ & $\beta=442$ \\
\hline
\end{tabular}

\subsection{Constructions from groups of order 38} $([7])$ :

The possible weight enumerators for self-dual codes of parameters $[78,39,14]$ are given as follows

$$
\begin{gathered}
W_{78,1}=1+(3705+8 \beta) y^{14}+(62244+512 \alpha-24 \beta) y^{16}+\ldots, 0 \leq \alpha \leq \frac{-1}{16} \beta \leq 28 \\
W_{78,2}=1+(3750+8 \alpha) y^{14}+(71460-24 \alpha) y^{16}+\ldots,-486 \leq \alpha \leq-135 .
\end{gathered}
$$

For many of these parameters, the existence of a code with that weight enumerator is not known. Together with the ones that were recently found in $[1,21,22]$, the existence of codes which have $W_{78,1}$ with $\alpha=0$ and $\beta=0,-13,-19-26,-38,-39,-52,-65,-78,-104,-117$ and which have $\alpha=-135$ in $W_{78,2}$.

In the following table we construct $[78,39,14]$ self-dual codes from $D_{38}$. The one with $\beta=-57$ is a new code. 
Table 22. Extremal $[78,39,14]$ codes from $D_{38}$ where $\left(\alpha_{1}, \alpha_{2}, \alpha_{3}, \alpha_{4}\right)=(1,0,1,1)$ and $a_{1}=a_{2}=a_{3}=$ $a_{4}=0$

\begin{tabular}{ccc}
\hline$\left(a_{5}, \ldots, a_{34}\right)$ & Aut $(C) \mid$ & $W_{78,1}(\alpha=0)$ \\
\hline$(1,1,1,1,0,1,0,1,1,0,0,1,0,1,0,1,0,0,0,0,0,0,1,1,1,1,1,1,0,1,1,1,1,1)$ & $2 \cdot 19$ & $\beta=0$ \\
\hline$(1,1,1,1,0,1,0,1,0,0,0,1,0,1,1,0,0,0,1,0,1,1,0,0,1,1,1,1,0,1,1,1,1,1)$ & 19 & $\beta=0$ \\
\hline$(1,1,1,1,0,1,0,0,0,0,1,1,0,0,0,1,0,0,1,0,1,1,0,0,0,1,1,0,1,0,1,0,1,1)$ & 19 & $\beta=-19$ \\
\hline$(1,1,1,1,0,0,1,0,1,1,0,0,0,0,0,0,0,1,0,1,1,0,0,1,1,0,1,1,0,1,0,0,1,1)$ & 19 & $\beta=-38$ \\
\hline$(1,1,1,1,0,0,1,1,1,0,0,1,1,1,0,1,0,0,1,0,0,0,1,0,0,0,0,0,0,1,1,0,1,1)$ & 19 & $\beta=-57$ \\
\hline
\end{tabular}

\section{Conclusion}

We have integrated a modified bordered construction with the matrices corresponding to a group ring element in $R C_{2 p}$ and $R D_{2 p}$ where $R$ is a commutative Frobenius ring of characteristic 2 and as a result we have been able to obtain many extremal binary self-dual codes. The structure of the groups has allowed us to look at such lengths as $62,70,78$, etc. that are different than the oft-studied lengths of 64 , 66 and 68 . In addition to giving an alternative construction to many extremal or best-known self-dual codes we were able to obtain a new code of length 78 , with $\alpha=0, \beta=-57$ in $W_{78,1}$. The results we have obtained demonstrate the relevance of our constructions and may lead to more such results when considered over different rings and groups.

Acknowledgment: The authors would like to thank the anonymous referees for their useful comments that helped improve the paper.

\section{References}

[1] D. Anev, N. Yankov, Self-dual codes of length 78 with an automorphism of order 13, in XVth International Workshop on Optimal Codes and Related Topics, Sofia, Bulgaria, July 2017.

[2] F. Bernhardt, P. Landrock, O. Manz, The extended Golay codes considered as ideals, J. Combin. Theory Ser. A 55(2) (1990) 235-246.

[3] J. H. Conway, N. J. A. Sloane, A new upper bound on the minimal distance of self-dual codes, IEEE Trans. Inform. Theory 36(6) (1990) 1319-1333.

[4] R. Dontcheva, New binary [70, 35, 12] self-dual and binary [72, 36, 12] self-dual doubly-even codes, Serdica Mathematical Journal 24(4) (2001) 287-302.

[5] S. T. Dougherty, J. Gildea, R. Taylor, A. Tylshchak, Group rings, G-Codes and constructions of self-dual and formally self-dual codes, Des. Codes Crypt. 86(9) (2018) 2115-2138.

[6] S. T. Dougherty, P. Gaborit, M. Harada, P. Sole, Type II codes over $\mathbb{F}_{2}+u \mathbb{F}_{2}$, IEEE Trans. Inform. Theory 45 (1999) 32-45.

[7] S. T. Dougherty, M. Harda, T. A. Gulliver, Extremal binary self-dual codes, IEEE Trans. Inform. Theory 43(6) (1997) 2036-2047.

[8] S. T. Dougherty, B. Yildiz, S. Karadeniz, Codes over $R_{k}$, Gray maps and their binary images, Finite Fields Appl. 17(3) (2011) 205-219.

[9] S. T. Dougherty, B. Yildiz, S. Karadeniz, Self-dual codes over $R_{k}$ and binary self-dual codes, Eur. J. Pure Appl. Mathematics 6(1) (2013) 89-106.

[10] P. Gaborit, V. Pless, P. Sole, O. Atkin, Type II codes over $\mathbb{F}_{4}$, Finite Fields Appl. 8(2) (2002) $171-183$. 
[11] J. Gildea, A. Kaya, R. Taylor, B. Yildiz, Constructions for self-dual codes induced from group rings, Finite Fields Appl. 51 (2018) 71-92.

[12] M. Gurel, N. Yankov, Self-dual codes with an automophism of order 17, Mathematical Commun. 21(1) (2016) 97-107.

[13] M. Harada, The existence of a self-dual [70,35, 12] code and formally self-dual codes, Finite Fields Appl. 3(2) (1997) 131-139.

[14] M. Harada, K. Saito, Singly even self-dual codes constructed from Hadamard matrices of order 28, Australas. J. Combin. 70 (2018) 288-296.

[15] T. Hurley, Group rings and rings of matrices, Int. J. Pure and Appl. Math. 31(3) (2006) 319-335.

[16] T. Hurley, Self-dual, dual-containing and related quantum codes from group rings, arXiv:0711.3983, 2007.

[17] S. Ling, P. Sole, Type II codes over $\mathbb{F}_{4}+u \mathbb{F}_{4}$ ", Europ. J. Combinatorics 22 (2001) 983-997.

[18] I. McLoughlin, A group ring construction of the [48,24,12] Type II linear block code, Des. Codes Crypt. 63(1) (2012) 29-41.

[19] I. McLoughlin, T. Hurley, A group ring construction of the extended binary Golay code, IEEE Trans. Inform. Theory 54(9) (2008) 4381-4383.

[20] E. M. Rains, Shadow bounds for self-dual codes, IEEE Trans. Inform. Theory 44 (1998) 134-139.

[21] N. Yankov, D. Anev, M. Gurel, Self-dual codes with an automorphism of order 13, Adv. Math. Commun. 11(3) (2017) 635-645.

[22] T. Zhang, J. Michel, T. Feng, G. Ge, On the existence of certain optimal self-dual codes with lengths between 74 and 116, The Electronic Journal of Combinatorics 22(4) (2015) 1-25. 